\title{
L’Église catholique a-t-elle subi des modifications en venant en Nouvelle-France?
}

\section{Georges-Émile Giguère}

Volume 15, numéro 2, septembre 1961

URI : https://id.erudit.org/iderudit/302111ar

DOI : https://doi.org/10.7202/302111ar

Aller au sommaire du numéro

Éditeur(s)

Institut d'histoire de l'Amérique française

ISSN

0035-2357 (imprimé)

1492-1383 (numérique)

Découvrir la revue

Citer cet article

Giguère, G.-É. (1961). L'Église catholique a-t-elle subi des modifications en venant en Nouvelle-France ? Revue d'histoire de l'Amérique française, 15(2), 189-203. https://doi.org/10.7202/302111ar d'utilisation que vous pouvez consulter en ligne.

https://apropos.erudit.org/fr/usagers/politique-dutilisation/ 


\section{L'ÉGLISE CATHOLIQUE A-T-ELLE SUBI DES MODIFICATIONS EN VENANT EN NOUVELLE-FRANCE ? *}

A notre connaissance, les historiens catholiques de langue française du Canada ne se sont jamais demandé si leur Église avait subi des modifications en venant en Nouvelle-France au XVII ${ }^{e}$ siècle. Spontanément ils n'ont pas de raisons de s'en inquiéter. Ma première inquiétude sérieuse à ce sujet m'est venue quand le Dr Walsh de l'Université McGill m'a posé la question cet automne en me demandant d'y répondre.

Plusieurs historiens anglo-canadiens, ${ }^{1}$ au contraire, ont scruté le problème et en ont même établi des éléments fort intéressants que nous allons utiliser en les replaçant dans un cadre d'ensemble. Je dois remercier très sincèrement le Dr Walsh pour son intrigante question et pour l'invitation qu'il m'a faite. C'est avec grand plaisir que je réponds à l'une et à l'autre aujourd'hui. Je désire particulièrement faire de ce travail une façon personnelle de commémorer l'arrivée des Jésuites qui apportaient l'Église catholique en Nouvelle-France, il y a 350 ans (1611).

En résumé, ma réponse peut être celle-ci : l'Église catholique du XVII ${ }^{e}$ siècle n'a pas subi de modifications substantielles en venant en Nouvelle-France. Cependant ce serait un non-sens, une invraisemblance de soutenir qu'elle n'a pas pris le visage de la Nouvelle-France et de tout son monde contemporain. Mais ces modifications sont accidentelles. L'Église est une société divine sujette aux conditionnements des circonstances humaines et tem-

* Conférence prononcée le 18 mai 1961, lors de la réunion annuelle des Religious Learned Societies, devant les membres de la Church Historical Society, à l'invitation spéciale du Dr N. H. Walsh de la Faculty of Divinity de l'Université McGill.

1 Il faut mentionner ici les noms de R. A. M. Lower, Edgar McInnis, J. M. S. Careless et l'historien américain Mason Wade que nous citerons par la suite. 
porelles. En conséquence, elle est inévitablement marquée par les hommes qui la composent ou la dirigent comme par l'époque où elle évolue.

\section{PAS DE MODIFICATIONS SUBSTANTIELLES}

Les modifications substantielles toucheraient son dogme (Credo), sa morale, son droit (ecclésiastique ou canonique) et peut-être ses cadres administratifs. Ces différents aspects sont ses principes de base, ses éléments d'unité, la source de sa force et ils sont tous placés sous la garde et vigilance du Souverain Pontife, son chef et son guide. En dehors de l'Église enseignante, soit le Pape et les évêques réunis en concile, vouloir changer ces points capitaux, c'est vouloir couper le rameau du grand arbre, séparer l'Église de la Nouvelle-France de l'Église catholique. Ce serait encore plus grave à nos yeux que de vouloir créer une Église nationale, ce serait créer une autre Église.

Into Canada, écrit Lower, 'ecclesiastical Gallicanism' did not enter: there were no disputes in the colony as to where lay the ultimate power to shape Catholic doctrine; in fact, there were no doctrinal disputes whatever. ${ }^{2}$

Il ne faut pas voir là matière à reproche pour une situation quasi anormale attribuable aux pressions de l'autorité locale, mais plutôt un signe d'unité vu que l'Église de la Nouvelle-France était simplement au diapason de l'Église universelle.

Thanks to Laval and the Jesuits, reprend Lower, a completely orthodox, wholly ultramontane Catholicism was nurtured in New France and to this day there have been no departures from it. ${ }^{3}$

Il faut remarquer que l'époque des grandes disputes théologiques est terminée en Europe. L'Église catholique de NouvelleFrance ne se distingue donc pas, vu que depuis un siècle Rome a défini et affirmé ses positions morales et disciplinaires au Concile de Trente (XVI ${ }^{\mathrm{e}}$ siècle). Ces faits s'éclaireront avec la suite de notre exposé. Loin de nous cependant de vouloir minimiser

2 A. R. M. Lower, Canadians in the Making (Toronto, 1958), 65.

3 Lower, op. cit., 65. 
le très grand effort de compréhension d'un historien comme Lower qui a voulu au surplus y mettre toute sa bienveillance en même temps que d'infinies précautions. "He who would understand the intellectual system upon which a Catholic community turns, must understand its philosophical foundations $\gg,{ }^{4}$ préciset-il.

C'est pour cette raison précise que le même historien s'empresse d'écrire: «A word, then, on the medieval background ${ }^{5}$ après avoir affirmé: «No branch of the Roman Catholic Church has more faithfully preserved the spirit of the high Middle Ages than that of French Canada. ${ }^{6}$

Pour bien montrer que Lower avait raison d'être circonspect, nous ajouterons le témoignage du R.P. Louis-M. Régis, dominicain, sur le même sujet.

La physionomie religieuse et philosophique du Canada français est toujours une énigme pour l'étranger, c'est-à-dire tant pour nos compatriotes de langue anglaise que pour les voyageurs et les immigrants qui essaient d'entrer en contact avec notre mode de penser et d'en avoir une conception précise. Elle est d'ailleurs, très souvent, un casse-tête chinois pour les Canadiens français eux-mêmes qui, au contact constant d'individus possédant d'autres habitudes de pensée et un autre idéal de vie, deviennent hésitants et perplexes au sujet des valeurs traditionnelles qui ont inspiré et guidé leur jeunesse et qui semblent avoir perdu leur signification et conséquemment leur efficacité au sein de la société qu'ils fréquentent. ${ }^{\top}$

Nous devons nous contenter à regret de cet extrait d'un texte qui éclaire si bien nos deux optiques. A son propos, Albert Lévesque a résumé dans une phrase nos positions de base respectives.

Le théologien catholique met l'accent sur le dogme (Credo), tandis que le théologien protestant

${ }^{4}$ Lower, op. cit., 56.

5 Lower, op. cit., 56.

${ }^{6}$ Lower, op. cit., 56.

7 Mason Wade, ed., Canadian Dualism / La Dualité canadienne (Toronto, 1960) ; Louis-Marie Régis, « La religion et la philosophie au Canada français », 56 . 
s'adonne plutôt à l'étude des langues originelles de l'Ancien et du Nouveau Testament. Quant à la philosophie, celle des catholiques est étroitement liée à la théologie; la Somme de saint Thomas semble leur suffire. Chez les Protestants 'the aim is not so much to supply the student with a carefull co-ordinated master plan of thought as to teach him to approach all philosophers with a keenly analytical mind and ultimately to achieve his own personal metaphysic to his own temperament - whether realistic, idealistic or pragmatic'. ${ }^{8}$

Voilà pourquoi nous pouvons conclure avec Lower qui écrit très justement: «It is much easier to describe the physical and corporate conditions of a society than its spirit. » 9

L'historien Lower ne renonce pas à une tâche qu'il estime pourtant difficile et il s'en acquitte même avec un brillant succès.

Equally imperative for study of the colonial Church is it to know a little about the major forces that at that time were affecting Catholic life, mainly, of course, in the motherland - Gallicanism, Jansenism, Jesuitism ... French Reformation ... ChurchState relationships. ${ }^{10}$

\section{MAIS DES MODIFICATIONS ACCIDENTELLES}

Les modifications accidentelles, avons-nous laissé entendre, tiennent surtout aux personnes, aux conditions sociales, économiques ou autres. A vrai dire, elles ne sont des modifications qu'en apparence puisqu'elles résultent de l'adaptation aux circonstances. Or on sait et il est abondamment démontré que la Nouvelle-France avait eu le temps de se différencier de la métropole française en plus d'un point. Le régime seigneurial, l'absence presque totale de classes sociales, le droit civil et le droit paroissial, le commerce des pelleteries et les procédés de guerre en fournissent autant de preuves. Lower l'a exprimé parfaitement par le sous-titre d'un de ses chapitres : «New France, not a replica of old ». ${ }^{11}$

8 Albert Lévesque, recension de l'ouvrage précédent dans Revue d'histoire de l'A mérique française, (mars 1961): 611.

9 Lower, op. cit., 48.

10 Lower, op. cit., 60.

11 Lower, op. cit., 28. 
Pour faire mieux saisir notre pensée sur ce point, nous empruntons quelques passages à l'historien Frégault:

Une société est avant tout un système de forces orientées dans un sens déterminé; découvrir le sens de ces forces, ce n'est pas seulement en indiquer la direction mais aussi en marquer la signification... (169). La société canadienne, on doit le reconnaître tout de suite, restait marquée d'hérédités françaises. Comment en eût-il été autrement ? Toute la politique de la colonie était non seulement inspirée, mais dirigée par la métropole; et l'ordre politique exerce nécessairement de grandes répercussions sur l'ordre social. Le Canada n'avait de contacts suivis et réguliers qu'avec la France. Les étrangers - particulièrement les Anglais, avec qui les Canadiens auraient pu entretenir des relations de voisinage - se voyaient fermer les portes du pays, en même temps que les Canadiens recevaient l'interdiction absolue de visiter les colonies anglaises... (170).

Qu'il y eut en Nouvelle-France des privilèges attachés à tel groupe, à telle classe ou à telles fonctions et que, d'autre part, le milieu rendît souvent ces privilèges illusoires, il n'est que d'observer, pour s'en convaincre, avec quelle application pointilleuse les individus et les groupes s'efforçaient de faire respecter les droits, les prérogatives et les avantages, souvent infimes, que leur assurait l'ordre social... (171).

Les querelles de préséance étaient si vives et si fréquentes que le gouvernement métropolitain jugea indispensable d'intervenir. Bien que réglés par de multiples ordonnances particulières, les honneurs réservés aux divers fonctionnaires coloniaux dans les églises provoquaient une infinité de contestations qui avaient parfois un invraisemblable retentissement... (174). On prenait au sérieux sa propre dignité et l'on n'entendait pas badiner sur ce point... (176).

Cet ensemble de petites distinctions honorifiques semble moins important que pittoresque. Cependant il convient de voir, à travers les manies amusantes de tout petits personnages, quelque chose qui 
va beaucoup plus loin. L'esprit d'une époque y transparaît. Ces détails éclairent un aspect capital de la société de la Nouvelle-France... (178).

En voilà suffisamment, croyons-nous, pour démontrer jusqu'à quel point la Nouvelle-France était soumise aux conventions sociales et avec quelle rigueur les rapports étaient réglés entre les divers éléments de sa population (179). ${ }^{12}$

En conséquence, on comprendra pourquoi nous reprendrons les grandes lignes de cette histoire. Cela nous permettra de signaler certains facteurs non négligeables, comme les rapports Église-État, ceux de la métropole avec sa colonie tant en matière politique que religieuse. A eux seuls ils fournissent un éclairage suffisant.

\section{ENTREPRISE PRIVÉE ET ENTREPRISE D'ÉTAT}

Nous devons faire observer d'abord que les Français se sont établis en Nouvelle-France avant la France. Par là nous voulons dire que les débuts ont été marqués par l'entreprise privée tant dans le domaine temporel que dans le domaine religieux. Au temporel, c'est le régime des compagnies qui a prévalu de 1603 à 1663. Durant soixante ans, il n'est pas question d'État, ni de ses fonctionnaires ni de ses fonds publics. Au point de vue religieux, c'est pour ainsi dire la même chose. Les communautés religieuses desservirent la colonie française et s'adornèrent au travail missionnaire, avant que l'Église ne fonde sa hiérarchie et ne crée un diocèse. Les missionnaires ont préparé le terrain, établi les premiers contacts, créé les premières œuvres d'évangélisation, d'enseignement et d'hospitalisation.

Cette première remarque est importante puisqu'en l'absence de l'État et même de l'Église, il ne peut être question de relations Église-État. Pour l'histoire ecclésiastique autant que pour l'histoire civile, il faut nécessairement distinguer la période antérieure à la colonie royale puisqu'elle est marquée de la meilleure collaboration entre missionnaires et administrateurs.

${ }^{12}$ Guy Frégault, La Civilisation de la Nouvelle-France (Montréal, 1944), loc. cit. 


\section{Les Huguenots CONTRE LE ROI ET CONTRE L'ÉGLISE}

De 1611 à 1629 , on voit apparaître progressivement l'Église par la présence des missionnaires. Après un bref mais pénible effort, les Jésuites Biard et Massé doivent quitter l'Acadie (16111612). Ce n'est pas avant 1615 que viennent à Québec les Récollets sur l'invitation explicite de Champlain. Ils travaillent seuls pendant dix ans avant de suggérer à Champlain de faire venir des Jésuites qui se mettent résolument à la tâche dès 1625. Pour tous ces missionnaires qui pensent avec l'Église, le travail de la grâce, de la surnature ne vient qu'après l'établissement des conditions naturelles. Mais là les missionnaires ont vu parfois leurs efforts paralysés.

Les compagnies marchandes, on s'en souvient, ont connu une existence précaire marquée de rivalités, de contestations et de suppressions. Assez ordinairement administrées par des Huguenots, elles étaient tenaces dans leurs revendications aussi bien marchandes que religieuses. L'État leur concédait le monopole de la traite et du commerce colonial et exigeait d'elles qu'elles transportent des colons et qu'elles établissent la religion catholique. Mais ces compagnies ont toujours pris uniquement les avantages économiques pour laisser tomber complètement leurs devoirs. De 1608 à 1624, Champlain lutta contre elles et dut multiplier ses voyages transocéaniques pour assurer la stabilité des compagnies et par elles l'établissement des colons.

En France, on venait à peine de sortir des guerres de religion. Richelieu n'a pas tardé à comprendre que les Huguenots français profitaient des largesses de l'Édit de Nantes pour faire obstacle à l'ascension de la monarchie absolue. De son côté, Champlain a suffisamment d'expérience pour inspirer au grand ministre une politique coloniale efficace. Richelieu, on lui en fait même le reproche, tout en étant cardinal de l'Église, fut d'abord un homme d'État, ${ }^{13}$ absolument fidèle à son roi. Dès son acces-

\footnotetext{
${ }^{13}$ Lower, op. cit., 21 . «It is true that he was a cardinal of the Church, but religious zeal did not play much part in that cold and opportunist heart. Primarily he was a stateman, playing the stateman's game power, and since considerations of power made it wise for him to favour Protestant countries, favour then he did. Hence his nickname, 'The Cardinal of the Huguenots'.»
} 
sion au pouvoir comme grand ministre (1624), il constate que les Huguenots divisent toujours la France. De plus il sait qu'ils se sont montrés encore plus mauvais colonisateurs en Floride et au Brésil qu'en Nouvelle-France. ${ }^{14}$ De plus, dans la colonie comme dans la métropole, ils ambitionnent un peu trop le monopole commercial et religieux. Ils soignent leurs intérêts et négligent leurs devoirs. Ils s'insurgent contre l'État.

Une nouvelle compagnie marchande, les Cent Associés, plus largement appuyée par l'État et contrôlée par lui, règlera le problème colonial. Au moment où le cardinal signe la charte de la dite compagnie (1627), il dirige le siège de LaRochelle, capitale des Huguenots et principal foyer de résistance à la souveraineté absolue du roi. Doit-on se surprendre qu'il ait exclu les Huguenots de la Nouvelle-France quand, bientôt, à la paix d'Alès (1629), il croira bon de leur retirer les avantages politiques et militaires qu'ils utilisent si mal ?

Avait-il tort d'ailleurs, puisqu'au dire de certains historiens, l'opération des frères Kirke contre Québec (1628-1629) est une entreprise huguenote au compte de l'Angleterre qui vient ellemême de participer à la résistance rocheloise contre l'autorité française ? Or la capitulation aux mains des Kirke met fin aux débuts prometteurs de la Compagnie des Cent Associés et à la Nouvelle-France elle-même.

$\mathrm{Au}$ retour de la France en Amérique en 1632, les Jésuites sont les seuls prêtres et religieux à revenir, vu que les Récollets ont décliné l'offre à cause du manque de sujets. Les Jésuites sont donc l'autorité religieuse. Leur effort sera considérable, total. On dira même qu'ils ont fait la Nouvelle-France: « Jesuit influence coloured the Church life of the colony. ${ }^{15}$ Pas d'émule, pas de compétition, pas de lutte. Et l'État n'est pas encore établi. On parle même parfois de théocratie des Jésuites, de Jésuitisme.

Durant cette période (1632-1663), plus encore que précédemment, la collaboration entre missionnaires et administrateurs

${ }^{14}$ Les Français en Amérique pendant la deuxième moitié du XVIe siècle. I - Les Français au Brésil, II - Les Français en Floride (Presses universitaires de France, Paris, 1946).

15 Lower, op. cit., 62. 
fut remarquable. On a déjà vu les missionnaires renseigner Champlain sur les mœurs indiennes et mettre au point sa politique coloniale et indigène. Et puis est-ce pure coïncidence si les compagnies deviennent plus stables après la venue des missionnaires. Deux historiens français, Salone et Goyau, ont établi la thèse que, sans l'adjonction de l'idée missionnaire à l'idée colonisatrice faite par les Jésuites, la Nouvelle-France n'aurait pas été possible. ${ }^{16}$ En instituant leurs Relations de la Nouvelle-France qu'ils distribuent annuellement à leurs amis, les Jésuites ont mis sur pied un puissant moyen de propagande au profit de la colonie. ${ }^{17}$ Ils fondent aussi des postes de missions, établissent le premier collège secondaire en Amérique du Nord. Ils attirent les communautés féminines pour l'éducation des filles et pour l'œuvre hospitalière. ${ }^{18}$

$\mathrm{Du}$ point de vue strictement religieux, cette époque mérite le nom d'Épopée mystique. ${ }^{19}$ Le vrai caractère de cette période dépasse les simples faits chronologiques et les cadres visibles d'une société même religieuse. En France, il faut s'en souvenir, on est à l'ère du renouveau spirituel. Tous les missionnaires qui en proviennent sont des âmes d'élite. Pour comprendre cette époque, il faut nécessairement pénétrer dans les âmes, voir la doctrine et scruter la vie de ceux qui l'ont faite. C'est une période de pionniers, faite d'héroïsme, de dévouement et de sacrifices où le courage, loin d'enlever au réalisme de ses héros, provient précisément du fait qu'ils ne pouvaient pratiquement pas compter sur des appuis extérieurs suffisants. Francis Parkman en a été vivement frappé. ${ }^{20}$ Tout était un terrible défi qu'ils ont relevé victo-

16 Georges Goyau, Epopée mystique. Les Origines religieuses du Canada (Paris, 1924). Emile Salone, La Colonisation de la Nouvelle-France (Paris, 1908).

17 Lower, op. cit., 85. - Léon Pouliot, Les Relations des Jésuites de la Nouvelle-France (Paris, 1940). Edgar McInnis, Canada: A Political and social History (Toronto, 1954), 38 et 70.

${ }^{18}$ Léon Pouliot, Les Relations des Jésuites de la Nouvelle-France. Il faut noter ici que le Canada français se glorifie d'avoir compté les premières femmes missionnaires de l'histoire de l'Eglise.

19 Voir Goyau, Epopée mystique.

20 Francis Parkman, The Jesuits in New France. - McInnis, op. cit., 70: "It was the beginning of one of the most remarkable passages in the history of the Continent. » 
rieusement au prix de leur travail et de leur sang. Histoire certes unique au monde, mais qui perd de son intensité dès que les conditions matérielles s'améliorent. Voilà qui est conforme à une constante de l'histoire: dans la mesure où manquent les appuis temporels, les forces de l'esprit peuvent se manifester dans toute leur énergie et dans toute leur véritable splendeur.

Les Jésuites ont en quelque sorte préparé l'Église. Ils ont connu les besoins de cette petite communauté religieuse. Ils ont bientôt senti le besoin d'un évêque dans la jeune colonie. Une question de juridiction ecclésiastique est soulevée brusquement avec l'arrivée de l'abbé de Queylus. Le désir de promouvoir la colonie missionnaire dans le cadre hiérarchique de l'Église par la nomination d'un évêque tel que le préconisaient les Jésuites, finit par triompher. Mgr de Laval devient premier évêque du Canada en 1659.

Ceux qui ont travaillé à faire établir la hiérarchie ecclésiastique vont maintenant travailler à faire bâtir la hiérarchie civile. Voici ce qu'écrit le chanoine Lionel Groulx à ce propos :

Les Relations des Jésuites, le Père Paul LeJeune, alors procureur en France de la mission du Canada, en 1662, le Père Ragueneau, membre du Conseil de Québec, Mgr de Laval, de passage à Paris quelques semaines à l'automne de cette même année, ont plaidé, tour à tour, avec chaleur et sur le ton le plus suppliant, la cause de la colonie. D'autres ont parlé ferme: les gouverneurs d'Argenson et d'Avaugour... D'Avaugour est allé plus loin. Il a dépêché à Paris une sorte d'ambassadeur. Et, pour cette ambassade, il a choisi l'homme qui pouvait le mieux s'en acquitter: Pierre Boucher... ${ }^{21}$

L'État en Nouvelle-France: L'AbSolutisme Royal, le GalliCANISME ET L'ULTRAMONTANISME

Dans la colonie, la période de l'entreprise privée est maintenant terminée. C'est durant cette époque qu'il faut situer la question des Huguenots ou Calvinistes, de la Réforme française

${ }^{21}$ Lionel Groulx, Histoire du Canada français depuis la découverte, (4 vol., Montréal, 1950), I: 72. 
et de ce qu'on appelle le Jésuitisme. Nous croyons avoir répondu à ces différents points. L'année 1665 met subitement en présence l'Église et l'État en Nouvelle-France avec l'arrivée d'un triumvirat: Tracy, Courcelles et Talon. Encore là pour bien comprendre, il faut nécessairement évoquer l'arrière-plan français. On pourra alors mieux saisir le problème du Gallicanisme et de l'Ultramontanisme.

Les pages que Lower consacre à «the struggle for dominance in and over the Catholic Church of French Canada ${ }^{22}$ et conséquemment au Gallicanisme comptent parmi les meilleures de son ouvrage. Voici comment il expose le Gallicanisme.

Sometimes the king's attempt to control the Church is termed 'political Gallicanism', while the efforts of the Church of France to free itself from too much Roman control are known as 'ecclesiastical Gallicanism'. They both came out to the same thing, a national church, with the king as its secular head, just as in England. In the seventeenth century, only by the narrowest margins did Gallicanism miss being a repetition of Anglicanism. Louis XIV went a considerable distance in the footsteps of Henry VIII but by the chance of circumstances and a pious wife, he did not go the whole. With both monarchs it was much more a matter of political advantage than of religion. ${ }^{23}$

En 1661 commence le règne personnel de Louis XIV. Aidé de Colbert, le roi construit un État fort qu'il incarne lui-même («L'État, c'est moi !»). Il raffermit le pouvoir de la monarchie par la centralisation administrative et porte sa souveraineté au sommet de l'absolutisme. Le roi se mêle de tout, même de l'Église et de la religion.

In 'political Gallicanism', écrit Lower, the State eventually won a large victory. Despite Laval, the king, acting through governor, intendant, and law courts, came to control the ecclesiastical authorities at every point. But the road to victory ever rang with the noise of battle. Despatches to France constantly advise that it is time to institute a secular

22 Lower, op. cit., 62.

23 Lower, op. cit., 64. 
clergy ... In the end, church juridiction over lay persons was cut down to the lowest point, the civil power having the last word in every case... ${ }^{24}$

Gustave Lanctôt qui a étudié minutieusement l'administration en Nouvelle-France a écrit à plus d'une reprise: «L'Église canadienne était l'absolue prisonnière de l'État. ${ }^{25}$ Lower est plus nuancé quand il écrit:

In the ancien régime, Church and State were twisted up together. If at a given moment one appeared dominant, this reflected the accident of personnalities: there was no thought of revolutionary change in their relationships. It is not possible to make a simple statement to the effect that as the power of the Church fell, that of the State rose. ${ }^{26}$

On peut facilement imaginer que, dans un Etat absolu, Colbert était gallican et que Talon devait être gallican aussi. D'ailleurs la preuve en est faite aujourd'hui. ${ }^{27}$ Les Instructions de l'intendant lui enjoignaient précisément de mettre les Jésuites à leur place dès son arrivée. Il ne tarda pas à prendre des mesures parmi lesquelles on peut compter une enquête. Mais il dut conclure que les Jésuites ne faisaient pas interférence à l'État. Les Jésuites voulurent même que l'affaire fût portée devant les tribunaux pour se voir justifier publiquement. Talon refusa et se contenta de faire rapport au roi.

Frontenac, comme l'intendant, son prédécesseur, voulut prendre les Jésuites en défaut sur le commerce des pelleteries. ${ }^{28}$ Mais il n'eut pas plus de succès que Talon. Cette fois les Jésuites allèrent se justifier à Rome même. Frontenac se contenta de les calomnier dans ses lettres au roi. Dans la querelle de l'eau-de-vie, l'évêque et les Jésuites furent en butte au gouverneur Frontenac. Pour les mettre en échec, «the courtier governor » ${ }^{29}$ avait amené

\footnotetext{
${ }^{24}$ Lower, op. cit., 66. 41-3, 76.

25 Gustave Lanctôt, L'Administration de la Nouvelle-France (Paris),

26 Lower, op. cit., 64.

27 Joseph Cossette, s.j., « Jean Talon, champion au Canada, du gallicanisme royal, 1665-1672 », dans RHAF (déc. 1957), XI: 329-352; et Thomas Chapais, Jean Talon, intendant de la N.F. (Québec, 1911).

28 Jean Delanglez, Frontenac and the Jesuits (Chicago, 1939), 133 ss.

29 J. Eccles, Frontenac, the courtier governor.
} 
avec lui des Récollets parce que plus souples entre ses mains. Il discuta encore avec $\mathrm{M}^{\mathrm{gr}}$ de Laval la nomination aux cures qu'il voulait stables tandis que l'évêque voulait se réserver le droit de changer ses curés de paroisses. Malgré les ordres de Frontenac, les missionnaires soutinrent que la francisation des indiens était une impossibilité.

\section{LE JANSÉNISME}

Nous réglerons assez rapidement le cas du Jansénisme, car son existence en France se rattache assez intimement à l'histoire du Gallicanisme. On peut dire qu'il en est de même en NouvelleFrance. L'historien Frégault nous dit pourquoi: "Il va de soi que l'on rejette avec vigueur le jansénisme dans un pays si respectueux de l'autorité royale. Pour la même raison, il serait inconcevable que le gallicanisme ne montrât pas parfois le bout de l'oreille.» ${ }^{30}$ Frégault avait écrit une page plus haut: "Si l'autorité de l'évêque assure l'unité de direction, elle contribue en même temps à maintenir la pureté de la foi et l'intégrité de la doctrine catholique. $\mathrm{Au} \mathrm{XVIII}{ }^{\mathrm{e}}$ siècle, les hérétiques ne sont pas tolérés en Nouvelle-France. Il n'y viendra que deux jansénistes ... ${ }^{31}$

\section{L'ÉDUCATION}

Enfin venons-en à l'éducation puisqu'en Nouvelle-France elle se rattache intimement à l'Église. Careless l'a bien saisi comme Lower puisqu'il affirme nettement:

The Church also exercised power over men's minds through controlling teaching and the institutions of learning. The close connection between religion and education was, of course, a deeprooted Catholic idea, and it was not surprising that the Church, not the State, should found and direct schools in New France. Because of the wholly orthodox Catholic atmosphere in the colony, however, there was no development of learning apart from the Church, as in Old France. There was no secular

30 Guy Frégault, La Civilisation de la Nouvelle-France, 240.

31 Frégault, op. cit., 239. 
education, no attempt to inquire and certainly no attempt to criticize the authority of Church teachings...

Yet the ignorance among the masses was no worse than in many other countries of the age. And certainly the Church laboured hard to reduce it. Religious orders sought to establish schools as well as missions and hospitals, and several famous schools were founded that still endure...

Hence ignorance in New France did not follow from the Church's control of education. The nature of that education, however, theoretical and classical rather than practical or scientific, remained firmly fixed in French Canada, to affect the thinking and outlook of its people for centuries thereafter. ${ }^{32}$

Et nous dirions que cette pensée rencontre celle de Frégault qui, au terme d'une brève étude, concluait ses propos sur l'éducation:

Voilà donc la Nouvelle-France dotée d'un système complet d'instruction publique. Elle s'est donné à sa mesure et peut-être même un peu plus haut que sa taille. Ses institutions d'enseignement primaire sont aussi bien organisées que le permettent les cadres territoriaux; ses maisons d'enseignement secondaire ne cherchent qu'à s'étendre et à se multiplier; quant à son enseignement supérieur, il est encore en germe mais, eu égard aux circonstances, ce qui est étonnant, c'est qu'il ne soit pas tout à fait inexistant. - A qui doit-elle tout cela? A l'Eglise d'abord, à ses prêtres, à ses religieuses, à ses religieux enseignants qui, avec des moyens souvent très pauvres - il faut y insister - sont parvenus à bâtir de solides institutions parce qu'ils savaient voir grand malgré tout et exiger d'eux-mêmes leur plein rendement personnel et humain ... ${ }^{33}$

\section{CONCLUSION}

Nous ne voudrions pas terminer sans faire une dernière observation, car pour répondre à une partie de la question, nous 65-66.

32 J. M. S. Careless, Canada. A Story of Challenge (Cambridge, 1953), 33 Frégault, op. cit., 265. 
avons dû insister sur certains éléments de différenciation qui ont pu donner à la Nouvelle-France et à son Église un visage différent de celui de la France du XVI ${ }^{e}$ siècle. Nous en concluons que c'était affaire d'adaptation ou conséquence inévitable d'un contexte historique. Il est cependant un autre aspect que nous avons dû négliger. C'est l'œuvre constructive, l'immense tâche apostolique accomplie sur toute l'étendue de ce continent: missions auprès des indigènes, œuvres éducationnelles et hospitalières, et même œuvre scientifique dans différents domaines: explorations, géographie et cartographie, ethnologie et linguistique. Nous y aurions vu un autre aspect du visage de l'Église en terre d'Amérique. Nous aurions pu y constater que si les Français pouvaient se chicaner sur des questions de préséance ou de prestige, ils savaient également construire, même dans la souffrance et la privation, des œuvres dignes de notre admiration. C'est pourquoi toujours il reste vrai que le Canada trouve dans ces pages d'introduction, les plus belles de son Story of Challenge.

Georges-ÉmiLe GiguÈRe, s.j. 